\title{
Dias ao Parto de Fêmeas Nelore de um Experimento de Seleção para Crescimento. I - Modelo de Repetibilidade ${ }^{1}$
}

\author{
Maria Eugênia Zerlotti Mercadante 2, 4, Irineu Umberto Packer ${ }^{3}, 4$, Alexander George Razook 2, 4, \\ Joslaine Noely dos Santos Gonçalves Cyrillo², Leopoldo Andrade de Figueiredo ${ }^{2}$
}

RESUMO - Registros de datas de entrada na monta e respectiva data do parto, referentes a 1.247 fêmeas Nelore dos rebanhos experimentais da Estação Experimental de Zootecnia de Sertãozinho (IZ - SP), selecionadas para altos (seleção e tradicional) e para médios (controle) pesos ao sobreano foram usados para obter a variável dias ao parto, a fim de estudar o efeito da seleção para crescimento sobre o desempenho reprodutivo. Arquivos de novilhas e de vacas e novilhas foram analisados incluindo e não incluindo as não paridas. Nenhuma diferença significativa foi detectada entre os registros provenientes das vacas dos rebanhos selecionados e do controle, apesar das vacas do rebanho seleção apresentarem as maiores médias de dias ao parto na maioria dos arquivos estudados. Concordando com os resultados obtidos para o efeito de rebanho, o peso à seleção foi significativo somente para as vacas e novilhas, considerando as não paridas, com tendência das mais pesadas à seleção apresentarem menores valores para dias ao parto. Modelos nos quais não foi considerado o peso à seleção forneceram os mesmos resultados para o efeito de rebanho. As herdabilidades variaram de 0,02 a 0,16 , sendo as mais altas obtidas em arquivos nos quais foram incluídos os registros das não paridas, indicando que a observação de caracteres de reprodução somente das fêmeas férteis contribui para mascarar as diferenças genéticas entre os animais, e quando esta variabilidade é re-introduzida, designandose penalidades às fêmeas que não pariram, as diferenças genéticas entre os animais aparecem. Existem evidências que a seleção para peso não comprometeu o desempenho reprodutivo das fêmeas, mesmo sendo criadas em condições ambientais similares.

Palavras-chave: desempenho reprodutivo, gado de corte, matrizes bovinas, reprodução, seleção para peso ao sobreano

\section{Days to Calving of Nelore Cows From a Selection Experiment for Growth. I - Repeatability Model}

\begin{abstract}
Records of start of breeding season and subsequent calving, from 1,247 Nelore females raised at Sertãozinho Experimental Station (IZ - SP, Brazil), selected for high (selection and traditional lines) and for average (control line) yearling weights were used to compute days to calving and to study the effect of selection for yearling weight on reproductive performance. Data sets of heifers, and cow and heifers were analyzed with and without non-calvers females. There were no significant differences among the performance of females from selection lines (selection and traditional) and those control line (NeC), although days to calving were higher in the selection line for all data sets. According to the results for the selection line effect, the weight at selection was significant only for cows and heifers including non-calvers females, and those with higher weight at selection showed smaller values for days to calving. Models without weight at selection showed equivalent results for line. The heritabilities were between 0.02 and 0.16 , and the higher estimates were from data sets including non-calvers females, suggesting that the observation of reproductive traits only on fertile females can make-up the genetic differences between the animals and when this variability is re-introduced, assigning penalty for non-calvers females, the genetic differences appear. The results show that the selection for high yearling weight did not compromise female reproductive performance, even being raised under similar environmental conditions.
\end{abstract}

Key Words: reproductive performance, beef cattle, beef cows, reproduction, yearling weight selection

\section{Introdução}

O peso corporal ou a taxa de crescimento continua sendo o critério de seleção primário para a maioria dos criadores de bovinos de corte em todo o mundo, e de outras espécies exploradas para produção de carne. Em aves e suínos, que têm intervalos de gerações menores, os efeitos indesejáveis da seleção para peso corporal sobre o desempenho reprodutivo já são conhecidos (Rauw et al., 1998). Para bovinos de corte, até meados da década de 80 muito se discutiu sobre as conseqüências da seleção para peso corporal sobre a eficiência de produção, incluindo o desempenho reprodutivo das matrizes, principalmente

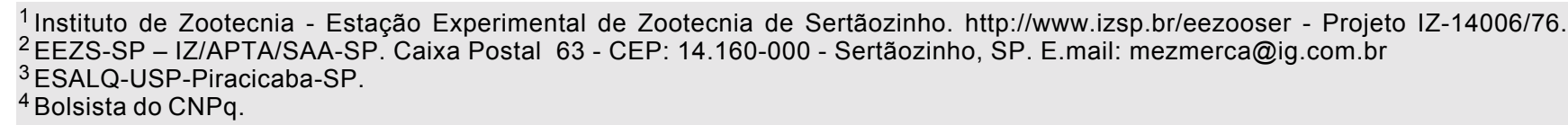


pelo aumento no peso adulto advindo dessa seleção (Koch et al., 1982; Scholtz \& Roux, 1984). Com o acúmulo dos dados dos experimentos de seleção por várias gerações, somado ao aumento quantitativo e qualitativo dos bancos de dados de criadores e associações de raças, e ao avanço dos métodos de predição, os estudos têm mostrado que a seleção para maior peso corporal não apresenta efeitos significativos sobre o desempenho reprodutivo das fêmeas (Wolfe et al., 1990; Meyer et al., 1991; Morris et al., 1992; Parnell, 1994; Archer et al., 1998; Mercadante et al., 2000; Silva et al., 2000;), embora na maioria deles os animais tenham sido criados em ambientes menos restritivos que o tropical.

A escolha do caráter reprodutivo a ser considerado tem variado entre autores. Dias ao parto (ou equivalentemente dia do parto ou data do parto) tem sido sugerido por alguns pesquisadores, enquanto outros têm argüido contra e defendido o uso da taxa de concepção ou parição(Ponzoni, 1992; Johnston \& Bunter, 1996). O caráter data do parto é realmente observado nas vacas paridas e reportado rotineiramente pelas associações de raça quando os bezerros são registrados. A expressão fenotípica de dias ao parto é composta, combinando efeitos do bezerro que controla o início do parto, efeitos da mãe associados com o início do ciclo estral e fertilidade, e os efeitos do touro em serviço associado com a qualidade do sêmen e da libido (Macneil \& Newman, 1994).

Apesar da limitação de um experimento de seleção em termos de número de animais, ele fornece excelente oportunidade de monitorar e estudar relações entre seleção direta e repostas correlacionadas. A Estação Experimental de Zootecnia de Sertãozinho (IZ - SP) iniciou, em 1976, um experimento de seleção, pioneiro em raças zebuínas, estabelecendo os rebanhos Nelore seleção e Nelore controle, a fim de examinar a resposta à seleção para peso corporal em raças de interesse nos trópicos, já que os poucos experimentos de seleção no mundo eram com raças européias. Hoje, após cerca de 4 gerações de seleção dessas populações, baseada em peso corporal, torna-se necessário estudar os efeitos no desempenho reprodutivo das fêmeas. Razook et al. (1998) relataram as respostas diretas no peso ao sobreano e as correlacionadas nos pesos ao nascer e ao desmame e Cyrillo et al. (2000), as respostas correlacionadas nas medidas corporais dos machos. $\mathrm{O}$ objetivo deste trabalho foi avaliar o efeito da seleção para peso ao sobreano no caráter dias ao parto, um dos indicadores do desempenho reprodutivo de fêmeas bovinas submetidas à estação de monta restrita.

\section{Material e Métodos}

\section{Animais e manejo}

As informações utilizadas para este estudo foram provenientes dos rebanhos da raça Nelore que fazem parte do projeto de seleção das raças zebuínas, estabelecido na Estação Experimental de Zootecnia de Sertãozinho (EEZS), unidade de pesquisa do Instituto de Zootecnia, da Secretaria de Agricultura e Abastecimento do Estado de São Paulo. Todos os detalhes da implantação dos rebanhos e do processo seletivo foram descritos por Razook et al. (1993, 1998).

Em linhas gerais, o experimento de seleção, iniciado em 1976, com a reorganização dos rebanhos e introdução de novas linhagens a partir do rebanho Nelore base existente na EEZS desde 1933, envolveu o estabelecimento de dois rebanhos, Nelore Controle $(\mathrm{NeC})$ e Nelore Seleção (NeS) em 1980. De 350 fêmeas aptas à reprodução, as 180 mais novas foram aleatoriamente designadas para os rebanhos Nelore Controle $(\mathrm{NeC})$ e Nelore Seleção, com 60 e 120 fêmeas respectivamente, e as 170 restantes, posteriormente formaram o rebanho Nelore Tradicional $(\mathrm{NeT})$. Foram usados anualmente 4,6 e até 8 touros nos rebanhos $\mathrm{NeC}, \mathrm{NeS}$ e NeT, sendo $50 \%$ deles com dois anos (primeira monta) e 50\% com três anos (segunda e última monta), selecionados de acordo com o diferencial de seleção, dentro de grupo contemporâneo rebanho $\mathrm{x}$ ano, para peso padronizado aos 378 dias (P378), obtido após prova de desempenho em confinamento de 168 dias de duração (Razook et al., 1997). As fêmeas de reposição foram selecionadas de acordo com o diferencial de seleção do peso padronizado aos 550 dias (P550), em regime de pasto, mas em geral foram retidas anualmente $50 \%$ delas para uma taxa anual de descarte de $20 \%$ das matrizes. Nos rebanhos selecionados $\mathrm{NeS}$ e NeT foram retidos para reprodução machos e fêmeas de maior diferencial e para o $\mathrm{NeC}$ aqueles com diferencial de seleção nulo. O NeT, em um esquema de reposição de touros e matrizes mais flexível, recebeu no início touros de fora dos rebanhos da estação, assim como, eventualmente, touros e matrizes descartados dos $\mathrm{NeC}$ e NeS. Após 15 anos (progênies de 1981 a 1995), passadas 3,5 gerações de seleção, com intervalo de gerações médio de 5 anos, os machos e as fêmeas do NeS têm, em média, 17 e $16 \%$ a mais no peso à seleção que os do $\mathrm{NeC}$ (Razook et al., 1998).

As novilhas de reposição foram colocadas em 
monta natural com dois anos de idade $(25,5 \pm 1$ meses), juntamente com o restante das fêmeas, em lotes médios de 25 matrizes para $\mathrm{NeS}$ e $\mathrm{NeT}$ e 15 matrizes para $\mathrm{NeC}$, para cada touro, durante 90 dias. Menores relações vaca/touro foram utilizadas para os tourinhos de 2 anos de idade, no primeiro ano de seu uso. Os bezerros foram desmamados nos meses de abril e maio, com 7 meses de idade. As fêmeas foram descartadas prioritariamente por falharem dois anos consecutivos, por problemas de saúde, por má habilidade materna (abandono de bezerro por dois anos consecutivos), ou por idade avançada, ao redor de 11 anos.

Dados

A variável dias ao parto (DIAP) foi obtida dos arquivos de estação de monta e parto dos anos de 1978 a 1998, com 7.336 registros de 1.425 fêmeas, filhas de 162 touros, para todas as fêmeas que pariram, a partir da diferença entre a data do parto e a data da entrada na estação de monta que deu origem a este parto. Na edição dos arquivos foram descartadas as informações provenientes de abortos, de partos fora da estação de nascimento (resultado de montas não controladas), e de inseminação artificial, totalizando 5.576 registros, pertencentes a 1.247 fêmeas. Durante os 20 anos, as datas de entrada e saída da monta foram 15 de novembro e 15 de fevereiro, com pequenas variações no decorrer dos anos. Em 5\% dos registros de parto, as vacas pariram após o início da estação de monta, e nestes casos a data de entrada na monta foi ajustada somando-se 4 dias à data do parto. Um sub-arquivo foi montado considerando somente os registros das fêmeas nascidas entre 1981 e 1996 $(\mathrm{n}=3.161)$, pois a partir de 1981 começaram a nascer as progênies dos rebanhos separados, e somente estas fêmeas têm registro do peso à seleção (P550). A partir deste arquivo, novilhas e vacas foram separadas, gerando dois arquivos distintos.

Após uma série de análises (Mixed; SAS, 2000) dos efeitos de rebanho de nascimento, ano do parto, ano de nascimento e touro em monta, o grupo contemporâneo para os registros repetidos de DIAP das vacas e vacas e novilhas foi definido como ano do parto $\mathrm{x}$ touro em monta. Nesta definição, o rebanho de nascimento não foi incluído pelo fato do $\mathrm{NeT}$ ter recebido, eventualmente, fêmeas descartadas dos rebanhos controle e seleção, ficando alguns grupos contemporâneos com reduzido número de observações. Para os registros de DIAP das novilhas, o grupo contemporâneo foi definido como ano x rebanho de nascimento, embora em muitas análises os efeitos foram considerados separadamente para testar a significância de rebanho. Neste arquivo não foi possível considerar, como no caso das vacas e vacas e novilhas, o efeito do touro em monta pelo fato das novilhas entrarem em monta com as vacas, sendo em pequeno número nos lotes de monta.

Provavelmente, as vacas vazias após o término da estação de monta são as de pior mérito genético para habilidade de conceber durante uma estação de monta restrita, e, portanto, o fato de ignorar estes animais pode ocasionar a exclusão das informações potencialmente mais valiosas sobre a diferença genética entre eles (Notter, 1988; Meyer et al., 1990). Johnston \& Bunter (1996) recomendaram somar 21 dias ao mais alto registro de DIAP dentro do grupo contemporâneo, após testar e discutir três métodos para designar um valor predito para as vacas não paridas. Dois dos métodos baseavam-se na soma de um valor fixo ao maior registro de DIAP dentro do grupo contemporâneo (21 ou 42 dias) e o terceiro, já usado anteriormente por Meyer et al. (1990), baseava-se na teoria de limiar para encontrar um só valor a ser designado aos registros de monta sem sucesso. Entretanto, o valor encontrado pode não ser mais alto que os valores mais altos dos registros válidos de DIAP pelo fato do método assumir distribuição normal, o que provavelmente não é o que ocorre e, assim, vacas paridas podem ser incorretamente comparadas às não paridas. Os autores ressaltam que pequena diferença foi observada entre os dois primeiros métodos ( 21 ou 42 dias), mas que considerando o método de 21 dias, os valores designados foram, em média, similares ao obtido a partir da teoria de limiar, com a vantagem de evitar o problema dos valores penalizados serem menores que os registros válidos.

Assim, a todo registro de monta sem DIAP foi designado um valor somando-se 21 dias ao maior registro de DIAP do grupo contemporâneo em que a fêmea estava (Johnston \& Bunter, 1996), penalizando as fêmeas que entraram na monta e não pariram. Para as novilhas este procedimento foi feito com os registros de primeira monta, e para as vacas e novilhas (nascidas de 1981 a 1996) separadamente das vacas e novilhas do arquivo completo (montas de 1978 a 1998 e fêmeas nascidas de 1961 a 1996). Este procedimento foi feito para todas as fêmeas que estavam em grupos contemporâneos com mais de 4 observações de DIAP. Pela ausência de diagnóstico

\footnotetext{
R. Bras. Zootec., v.31, n.4, p.1715-1725, 2002
} 
de prenhez, neste processo foram penalizadas também aquelas fêmeas que emprenharam e que tiveram aborto precoce não percebido pelo pessoal de campo. Foram penalizadas cerca de $25 \%$ das observações válidas de DIAP nos três arquivos descritos anteriormente.

Os dados foram analisados usando o procedimento para modelos lineares mistos (Mixed; SAS, 2000), a fim de testar os efeitos fixos e covariáveis e obter as médias ajustadas. Neste procedimento as médias de quadrados mínimos são provenientes da mesma matriz L usada para obtê-las no GLM, mas os erros-padrão são ajustados para os efeitos aleatórios do modelo (Littel et al., 1996). Os modelos ajustados incluíram, para novilhas, os efeitos fixos de rebanho e ano de nascimento (grupo contemporâneo), interação rebanho $\mathrm{x}$ ano de nascimento $\mathrm{e}$ as covariáveis exploratórias peso à seleção (P550) e idade na entrada da monta; e para as vacas e vacas e novilhas os efeitos de grupo contemporâneo (ano do parto + touro em monta), rebanho de nascimento, estado reprodutivo anterior, classe de idade da vaca na entrada da monta e as covariáveis P550 e peso na entrada da monta (PEM). Um modelo alternativo, considerando ano de nascimento e a interação rebanho $x$ ano de nascimento (e não mais idade na entrada da monta), foi ajustado para novilhas e vacas a fim de obter as médias ajustadas de DIAP por rebanho $\mathrm{x}$ ano de nascimento e as soluções de ano de nascimento para estimar a tendência ambiental. $\mathrm{Na}$ análise do arquivo completo, o P550 não foi considerado, pois só houve registro deste peso a partir das progênies de 1981, e para as vacas nascidas antes de 1981, antes da separação dos rebanhos, o rebanho de nascimento foi igualado ao rebanho de monta. $\mathrm{Na}$ análise de registros repetidos, a matriz de covariâncias residuais (R) ajustada para os registros de uma mesma vaca foi a auto-regressiva de primeira ordem, com homogeneidade de variâncias para os arquivos de vacas, vacas e novilhas sem as não paridas e para o arquivo completo, e com heterogeneidade de variâncias para o arquivo de vacas e novilhas incluindo as não paridas. Estas matrizes acomodam o padrão geral das correlações decrescendo com o aumento do intervalo entre as medidas, entretanto, este decréscimo é uniforme, diminuindo o número de parâmetros a serem estimados. A estrutura de R foi escolhida, dentre outras, com base no menor valor do critério de informação de Akaike (Littel et al., 1996).

Em todos os modelos descritos acima foram

R. Bras. Zootec., v.31, n.4, p.1715-1725, 2002 testados também os efeitos do sexo do bezerro, do coeficiente de endogamia e do coeficiente de geração (estes últimos foram incluídos ou um ou outro, como covariável), além de todas as interações possíveis, sendo em geral não significativos $(\mathrm{P}>0,05)$. Ajustando modelos com os mesmos efeitos fixos descritos acima, os componentes de variância genético aditivo (para novilhas, vacas e vacas e novilhas), permanente de ambiente (para vacas e vacas e novilhas) e residual foram estimados sob modelo animal no MTDFREML (Boldman et al., 1995). O arquivo de genealogia, com 5829 animais, foi estruturado considerando os pais das primeiras progênies (1978-1981) como fundadores. Para os arquivos das fêmeas nascidas a partir de 1981 e com registros penalizados, foram estimados os valores genéticos do DIAP da primeira monta (novilhas) e de todas as montas (vacas e novilhas).

\section{Resultados e Discussão}

\section{Os valores observados}

A média de DIAP, sem considerar as fêmeas não paridas, foi 328 dias, com coeficiente de variação muito baixo $(7,6 \%)$, pela própria definição do caráter, já que ele contém a gestação com até mais noventa dias da estação de monta. Considerando período de gestação médio em Nelore de 295 dias e coeficiente de variação de $2 \%$ (Pereira et al., 2000), o tempo médio pré-concepção na estação de monta (ou período de serviço) foi 34 dias, e as montas concentradas entre o primeiro e segundo terços da estação de monta, deslocando a curva de distribuição dos valores de DIAP para a esquerda (Figura 1). Com uma grande amostra de partos de fêmeas Nelore, Pereira et al. (2000) observaram média de 323 dias, com coeficiente de variação de 7,9\%. Nos arquivos incluindo as não paridas, a média e a variação (345 dias com coeficiente de variação de $11 \%$ ) foram logicamente maiores, pois foram somados 21 dias, introduzindo variabilidade e provocando um outro pico, menor que o dos registros observados, na curva de distribuição (Figura 1). Um problema que advém da própria definição do caráter dias ao parto é o valor mínimo considerado normal, que deve ser o menor valor aceitável para uma gestação normal. Ponzoni \& Gifford (1994) alertam que em um sistema de avaliação genética usando informações de vários parentes, gestações anormais curtas poderiam fornecer valores muito favoráveis para DIAP, afetando não só a 


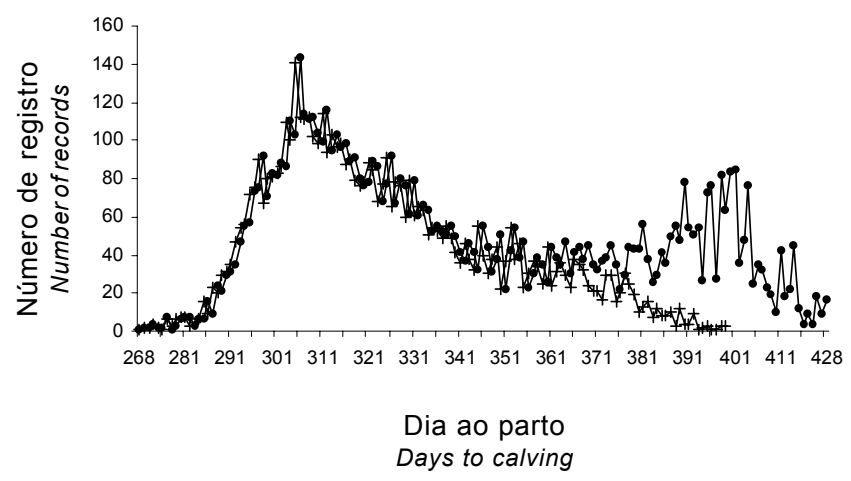

Figura 1 - Distribuição dos registros observados de dias ao parto, dos arquivos de novilhas e vacas, sem considerar $(+)$ e considerando as fêmeas não paridas $(\bullet)$.

Figure 1 - Distribution of days to calving records, from heifers and cows data set without (+) and with non-calvers (•) females.

estimativa do valor genético do próprio indivíduo, mas também a de seus parentes, incorretamente.

Uma das propriedades desejáveis atribuída aos dias ao parto, ou equivalentemente dia do parto, como variável reprodutiva, é a sua variação contínua, que contrasta com a natureza discreta (0 e 1) da variável alternativa sucesso ao parto (Ponzoni \& Gifford, 1994), entretanto, ela não apresenta distribuição normal (Figura 1). Meyer et al. (1990), Ponzoni \& Gifford (1994) e Gressler et al. (2000), utilizando transformações, falharam na tentativa de normalizar a variável, não obtendo efeito deste procedimento sobre os resultados obtidos. Em muitos outros estudos, entre eles os de MacNeil \& Newman (1994), Johnston \& Bunter (1996), Archer et al. (1998), Bergmann et al. (1998) e Pereira et al. (2000), nenhuma transformação foi feita e os registros de dias ao parto foram analisados na escala original, procedimento também adotado no presente estudo.

Fontes de variação

O grupo contemporâneo foi altamente significativo $(\mathrm{P}<0,01)$ tanto para as novilhas, para as vacas, como para as duas categorias juntas. A média de registros de DIAP por grupo contemporâneo foi 16, 10 e 12 nos arquivos de novilhas, vacas e vacas e novilhas, sendo que $21 \%, 45 \%$ e $28 \%$ dos registros ficaram em grupos com menos de 10 observações, respectivamente. As médias ajustadas de DIAP para os efeitos de rebanho de nascimento são apresentadas na Tabela 1.

\footnotetext{
R. Bras. Zootec., v.31, n.4, p.1715-1725, 2002
}

Orebanho de nascimento foi significativo $(\mathrm{P}<0,05)$ para as novilhas nos dois arquivos, sem considerar e considerando as fêmeas não paridas que tiveram o registro incluído por penalização, e, apesar do $\mathrm{NeS}$ apresentar sempre a maior média de DIAP, não houve diferença significativa entre os rebanhos selecionados $\mathrm{NeS}$ e $\mathrm{NeT}$ e o controle $(\mathrm{NeC})$. Novamente, mesmo sendo não significativo, para as vacas e vacas e novilhas (com exceção do arquivo completo) as médias do $\mathrm{NeS}$ foram mais altas em relação ao $\mathrm{NeC}$ e NeT, porém associadas a altos erros-padrão. Para o arquivo completo (incluindo todas as fêmeas participantes), $\mathrm{NeT}$ apresentou média maior e significativamente $(\mathrm{P}=0,05)$ diferente da média de $\mathrm{NeC}$, o que se explica pelo fato de ter sido o rebanho mais flexível na formação e na reposição das fêmeas, recebendo eventualmente as vacas descartadas dos rebanhos $\mathrm{NeS}$ e $\mathrm{NeC}$ e, possivelmente, menos férteis.

Os resultados com os arquivos incluindo as fêmeas não paridas podem ser considerados os mais importantes, funcionando como um tipo de análise da taxa de prenhez (Ponzoni, 1992; Johnston \& Bunter, 1996), uma vez que as fêmeas que foram para monta e não pariram em determinado ano receberam os mais altos valores de DIAP. A correlação genética de $-0,97$ entre dia do parto (incluindo as fêmeas não paridas) e sucesso ao parto (0 e 1), estimada por Johnston \& Bunter (1996), indica que eles são geneticamente o mesmo caráter, embora os autores atentem que a natureza binária do caráter sucesso ao parto não foi levada em conta e ele foi analisado por técnicas para caracteres com distribuição normal. Os resultados encontrados aqui mostraram que o desempenho das fêmeas selecionadas para maior peso aos 18 meses de idade (P550) não diferiu significativamente do desempenho daquelas selecionadas para a média de P550, sugerindo, indiretamente, taxas de prenhez semelhantes nos três rebanhos. Archer et al. (1998), analisando o desempenho reprodutivo das fêmeas do experimento de seleção com bovinos Angus na Austrália, não observaram diferenças significativas entre as selecionadas para maior ganho de peso diário e aquelas aleatoriamente selecionadas, tanto para dia do parto como para taxa de prenhez, apesar dos animais do rebanho selecionado apresentarem, em média, peso $14 \%$ acima da média dos animais do rebanho controle aos 365 dias de idade (Parnell et al., 1997). Os autores observaram ainda, períodos de gestação similares nas duas linhas seleção. Na análise dos caracteres de reprodução das 
Tabela 1 - Número de animais e de registros e médias ajustadas para dias ao parto ( \pm erro-padrão) para o efeito de rebanho

Table 1 - Number of animals and records and adjusted means for days to calving ( \pm standart error) for effects of selection line

\begin{tabular}{|c|c|c|c|c|c|c|}
\hline & $\begin{array}{c}\text { Novilhas } \\
\text { Heifers }\end{array}$ & $\begin{array}{l}\text { Novilhas com } \\
\text { as não paridas } \\
\text { Heifers with } \\
\text { non-calvers }\end{array}$ & $\begin{array}{l}\text { Vacas } \\
\text { Cows }\end{array}$ & $\begin{array}{c}\text { Novilhas } \\
\text { e vacas } \\
\text { Heifers and cows } \\
\text { with non-calvers }\end{array}$ & $\begin{array}{l}\text { Novilhas e vacas } \\
\text { com não paridas } \\
\text { Heifers and cows } \\
\text { with non-calvers } 1\end{array}$ & $\begin{array}{l}\text { Novilhas e vacas } \\
\text { com as não paridas } 1 \\
\text { Heifers and cows }\end{array}$ \\
\hline $\begin{array}{l}\text { Animais } \\
\text { Animals }\end{array}$ & 761 & 926 & 636 & 793 & 928 & 1406 \\
\hline $\begin{array}{l}\text { Registros } \\
\text { Records }\end{array}$ & 761 & 926 & 2359 & 3159 & 4212 & 7228 \\
\hline $\begin{array}{l}\text { Rebanho } \\
\text { Line }\end{array}$ & $\mathrm{P}=0,017$ & $\mathrm{P}=0,004$ & NS & NS & NS & $\mathrm{P}=0,0264$ \\
\hline $\mathrm{NeC}$ & $328 \pm 2^{\mathrm{ab}}$ & $347 \pm 3^{a b}$ & $315 \pm 7$ & $319 \pm 6$ & $335 \pm 9$ & $333 \pm 3^{a}$ \\
\hline $\begin{array}{l}\text { Control } \\
\mathrm{NeS}\end{array}$ & $333 \pm 1^{\mathrm{a}}$ & $354 \pm 2^{\mathrm{a}}$ & $332 \pm 5$ & $330 \pm 4$ & $340 \pm 6$ & $339 \pm 2^{\mathrm{ab}}$ \\
\hline $\begin{array}{l}\text { Selection } \\
\text { NeT } \\
\text { Traditional } \\
\end{array}$ & $328 \pm 1^{b}$ & $345 \pm 2^{b}$ & $323 \pm 3$ & $327 \pm 3$ & $338 \pm 4$ & $343 \pm 1^{b}$ \\
\hline
\end{tabular}

fêmeas do experimento de seleção com bovinos Angus na Nova Zelândia, Morris et al. (1992) também não encontraram diferenças significativas para porcentagem de vacas parindo e taxa de desmama das fêmeas selecionadas para maior peso a um ano de idade e daquelas aleatoriamente selecionadas. Neste experimento os animais da linha selecionada são, em média, $19 \%$ mais pesados a um ano de idade que aqueles do rebanho controle (Backer et al., 1991).

No presente trabalho, apesar dos rebanhos serem criados nas mesmas condições ambientais e de manejo, o tamanho dos lotes do $\mathrm{NeC}$ sempre foi menor que os dos rebanhos $\mathrm{NeS}$ e NeT, mas a dimensão dos piquetes de monta foi sempre a mesma, fato este que poderia ter favorecido as fêmeas do rebanho controle devido à maior disponibilidade de forragem durante 0 período de monta. Este efeito é levado em conta quando o touro em monta (ou equivalentemente o lote) foi incluído no grupo contemporâneo, que não foi feito para as novilhas devido ao reduzido número de observações nas subclasses.

As médias ajustadas de DIAP para os efeitos de prenhez anterior, classe de idade na entrada da monta, e os coeficientes de regressão para os efeitos de idade na entrada da monta, pesos à seleção e na entrada da monta são apresentadas na Tabela 2.

$\mathrm{O}$ peso à seleção (P550) só foi significativo $(\mathrm{P}<0,05)$ para os registros de vacas e novilhas considerando as fêmeas não paridas, concordando, de um modo geral, com os resultados obtidos para rebanho. $O$ coeficiente de regressão de P550 em relação a DIAP foi igual a $-0,07$, mostrando tendência das mais pesadas à seleção apresentarem valores mais baixos de DIAP. Tendência semelhante foi observada para o peso na entrada da monta (PEM), incluído nos modelos para vacas e vacas e novilhas, que, como esperado, esteve associado a menores DIAP.

Como a diferença entre o rebanho $\mathrm{NeC}$ e os selecionados ( $\mathrm{NeS}$ e NeT) está no P550, e, conseqüentemente nos pesos em idades posteriores, como os pesos na entrada da monta, uma série de análises foi realizada sem o P550 e o PEM. Para os registros das novilhas, modelos sem o efeito de rebanho forneceram os mesmos resultados para P550, o mesmo ocorreu no modelo sem P550, não alterando o resultado do efeito de rebanho, apesar das médias do DIAP de NeS serem sempre as mais altas. Para as vacas, e vacas e novilhas, no ajuste de modelos sem P550 e com PEM, de modelos com PEM e sem P550, e de modelos omitindo os dois pesos, o efeito de rebanho permaneceu não significativo. Retirando o efeito do PEM no modelo de análise de vacas e novilhas com as não paridas, o efeito do $\mathrm{P} 550$ permaneceu significativo $(\mathrm{P}<0,01)$, com um coeficiente de regressão mais alto $(-0,11 \pm 0,03)$.

Estas análises reafirmam os resultados obtidos (Tabela 1), sugerindo que não há diferenças no desempenho de DIAP nos rebanhos experimentais. 
Tabela 2 - Médias ajustadas de dias ao parto ( \pm erro-padrão) para os efeitos de prenhez anterior e classe de idade na entrada da monta, coeficientes de regressão ( \pm erro-padrão) para os efeitos de idade na entrada da monta, pesos à seleção (P550) e na entrada da monta (PEM), e variâncias genética aditiva, permanente de ambiente e residual, herdabilidade e repetibilidade

Table 2 - Adjusted means for days to calving ( \pm standart error) for effects of previous calving and class of age at start of breeding season, regression coefficients ( \pm standart error) for effects of the age at start of breeding season, weight at selection (P550) and at start of breeding season (PEM), and additive genetic, permanent and residual variances, heritability and repeatability

\begin{tabular}{|c|c|c|c|c|c|c|}
\hline & $\begin{array}{c}\text { Novilhas } \\
\text { Heifers }\end{array}$ & $\begin{array}{l}\text { Novilhas com } \\
\text { as não paridas } \\
\text { Heifers with } \\
\text { non-calvers } \\
\end{array}$ & $\begin{array}{l}\text { Vacas } \\
\text { Cows }\end{array}$ & $\begin{array}{c}\text { Novilhas } \\
\text { e vacas } \\
\text { Heifers and cows } \\
\text { with non-calvers }\end{array}$ & $\begin{array}{l}\text { Novilhas e vacas } \\
\text { com não paridas } \\
\text { Heifers and cows } \\
\text { with non-calvers } 1 \\
\end{array}$ & $\begin{array}{l}\text { Novilhas e vacas } \\
\text { com as não paridas } 1 \\
\text { Heifers and cows }\end{array}$ \\
\hline Prenhêz anterior & & & $\mathrm{P}<0,001$ & $\mathrm{P}<0,001$ & $\mathrm{P}<0,001$ & $\mathrm{P}<0,001$ \\
\hline \multicolumn{7}{|l|}{ Previous calving } \\
\hline Não (no) & & & $316 \pm 2$ & $317 \pm 1$ & $321 \pm 2$ & $322 \pm 1$ \\
\hline $\operatorname{Sim}(y e s)$ & & & $330 \pm 1$ & $334 \pm 1$ & $355 \pm 2$ & $354 \pm 1$ \\
\hline Idade monta & & & $\mathrm{P}<0,001$ & $\mathrm{P}<0,001$ & $\mathrm{P}<0,001$ & $\mathrm{P}<0,001$ \\
\hline \multicolumn{7}{|l|}{ Age breeding } \\
\hline 2 anos (years) & & & & $336 \pm 2^{a}$ & $354 \pm 3^{a}$ & $354 \pm 2^{\mathrm{a}}$ \\
\hline 3 anos & & & $331 \pm 2^{\mathrm{a}}$ & $330 \pm 1^{b}$ & $343 \pm 2^{b}$ & $343 \pm 1^{b}$ \\
\hline 4 anos & & & $324 \pm 1^{\mathrm{b}}$ & $325 \pm 1^{\mathrm{c}}$ & $336 \pm 2^{c}$ & $335 \pm 1^{\mathrm{c}}$ \\
\hline 5 anos & & & $319 \pm 1^{\mathrm{c}}$ & $319 \pm 1^{\mathrm{d}}$ & $328 \pm 2^{\mathrm{d}}$ & $329 \pm 1^{\text {de }}$ \\
\hline$>5$ anos & & & $319 \pm 1^{\mathrm{c}}$ & $318 \pm 1^{\mathrm{d}}$ & $328 \pm 2^{d}$ & $330 \pm 1^{\mathrm{e}}$ \\
\hline $\begin{array}{l}\text { Idade monta-dias } \\
\text { Age breeding-days }\end{array}$ & $\mathrm{P}<0,001$ & $\mathrm{P}<0,001$ & & & & \\
\hline Linear (linear) & $-0,05 \pm 0,01$ & $-0,23 \pm 0,04$ & & & & \\
\hline P550 (kg) & NS & NS & NS & NS & $\mathrm{P}=0,041$ & \\
\hline Linear(linear) & $-0,09 \pm 0,05$ & $-0,05 \pm 0,07$ & $0,04 \pm 0,03$ & $-0,02 \pm 0,02$ & $-0,07 \pm 0,03$ & \\
\hline $\operatorname{PEM}(\mathrm{kg})$ & & & $\mathrm{P}=0,037$ & $\mathrm{P}<0,001$ & $\mathrm{P}<0,001$ & $\mathrm{P}<0,001$ \\
\hline Linear(linear) & & & $-0,03 \pm 0,01$ & $-0,05 \pm 0,01$ & $-0,10 \pm 0,02$ & $-0,09 \pm 0,01$ \\
\hline$\sigma_{\mathrm{a}}^{2}$ & 41,03 & 85,53 & 40,36 & 8,86 & 146,74 & 199,14 \\
\hline$\sigma_{\mathrm{c}}^{2}{ }^{3}$ & & & 52,72 & 68,34 & 103,01 & 66,19 \\
\hline$\sigma_{\mathrm{e}}^{24}$ & 549,79 & 1063,84 & 403,27 & 442,70 & 953,21 & 991,92 \\
\hline $\mathrm{h}^{2} \pm \mathrm{ep}^{5}$ & $0,07 \pm 0,06$ & $0,07 \pm 0,06$ & $0,08 \pm 0,04$ & $0,02 \pm 0,02$ & $0,12 \pm 0,03$ & $0,16 \pm 0,02$ \\
\hline $\mathrm{r}^{6}$ & & & 0,19 & 0,15 & 0,21 & 0,21 \\
\hline
\end{tabular}

a b , c d médias na mesma coluna com diferentes letras são significativamente $(P<0,05)$ diferentes; 1 todas as vacas das estações de monta de 1978-1998; ${ }^{2}$ variância genética aditiva; ${ }^{3}$ variância devido ao efeito permanente de ambiente; ${ }^{4}$ variância residual; ${ }^{5}$ herdabilidade \pm erro-padrão, ${ }^{6}$ repetibilidade.

$a, b, c, d$ means in the same column, and within the same effect, with different superscript are significantly $(P<.05) ;{ }^{1}$ all cows from breeding seasons of 1978 1998; ${ }^{2}$ additive genetic variance; ${ }^{3}$ permanent environmental variance; ${ }^{4}$ residual variance; ${ }^{5}$ heritability \pm standart error; 6 repeatability.

Os trabalhos com dados de experimentos de seleção em bovinos de corte não têm encontrado diferenças significativas entre o desempenho reprodutivo das fêmeas dos rebanhos selecionados para maior crescimento e dos rebanhos não selecionados, qualquer que seja o caráter estudado (Wolfe et al., 1990; Morris, et al., 1992, Parnell, 1994; Archer et al., 1998). Herd (1995), analisando duas linhas divergentes (alto peso-HW e baixo peso-LW) do experimento com Angus na Austrália, observou que as vacas da linha HW apresentaram requerimento energético diário de mantença semelhante às vacas da linha LW. Isto é, apesar da diferença no peso corporal das vacas provenientes das duas linhas seleção (24\%), as vacas HW necessitaram cerca de $79 \%$ da energia metabolizável por dia requerida pelas vacas LW para manter cada quilograma de peso corporal. Isto se deve ao fato das vacas HW apresentarem mais gordura e menos proteína por unidade de peso vivo que as vacas LW, e o custo de manutenção de um quilograma de proteína é aproximadamente 1,05 megajoule de energia metabolizável, enquanto que o custo para manter um quilograma de gordura tende a ser negativo (-0,11 megajoule). Este fato pode ser uma explicação para o desempenho reprodutivo semelhante entre linhas seleção apesar da diferença nos caracteres de crescimento. Vale ressaltar que nenhum desses experimentos, com a manutenção de 
uma população controle, teve mais que 5 gerações de seleção avaliadas, o que pode não ter sido suficiente para que problemas reprodutivos se manifestassem.

Vacas que falharam no ano anterior, incluindo as novilhas (nos três arquivos de novilhas e vacas), emprenharam mais cedo na estação de monta que as paridas no ano anterior $(\mathrm{P}<0,01)$, concordando com os resultados de MacNeil \& Newman (1994), que analisaram os dias ao parto das fêmeas Hereford da "Linha 1 de Montana" e Meyer et al. (1990), com dados de fêmeas Angus, Hereford e cruzadas. Johnston \& Bunter (1996) não encontraram efeito do estado reprodutivo anterior no desempenho do caráter dia do parto trabalhando com grande número de registros de fêmeas Angus. Apenas para os registros das vacas, a interação estado reprodutivo anterior e classe de idade à monta foi significativa $(\mathrm{P}=0,003)$, com as fêmeas de 3 anos paridas no ano anterior apresentando maiores médias de DIAP que as também paridas anteriormente nas classes de 4,5 e 6 anos. Estas fêmeas ainda estão em crescimento e amamentando um bezerro e os aportes nutricionais podem não ter sido suficientes para crescimento e reprodução. Davis et al. (1993), trabalhando com o desempenho de fêmeas cruzadas de um experimento de seleção para taxa de prenhez, observaram efeito significativo desta interação, com vacas lactantes de segundo e terceiro partos parindo mais tarde.

A idade de entrada na estação de monta teve efeito significativo $(\mathrm{P}<0,01)$ nos registros de novilhas, apesar de todas as fêmeas terem sido expostas pela primeira vez ao redor de 24 meses de idade. Os registros originais, sem considerar as fêmeas não paridas, apresenta uma variação maior na idade de entrada na estação de monta (2,3 $\pm 0,4$ anos), pois são registros de primeiro parto e apresentam portanto partos resultantes de monta aos 2 anos e partos das que falharam no primeiro ano, resultantes de monta aos 3 anos de idade. Para os registros de DIAP resultantes de primeira monta e considerando as fêmeas que não pariram, a variação de idade é muito menor (2,1 $\pm 0,1$ anos). Como observado também em outros estudos (Rege \& Famula, 1993, Johnston \& Bunter, 1996, Archer et al., 1998, MacGregor \& Casey, 1999), para vacas e vacas e novilhas, a idade de entrada na monta foi significativa, com as médias de DIAP das fêmeas de dois e três anos, sendo mais altas que as demais. Nos arquivos de vacas e novilhas, incluindo as não paridas, novamente as maiores médias de DIAP são decorrentes de primeiro e segundo partos, podendo ser interpretadas como menores taxas de prenhez. Com o descarte das fêmeas inférteis, principalmente após a segunda monta, a taxa de prenhez aumenta, como mostram as médias mais baixas de DIAP nas classes 4,5 e 6 .

A expressão fenotípica dos dias ao parto é composta da combinação dos efeitos do bezerro controlando o início do parto, efeitos da mãe associados com o início do ciclo estral e fertilidade, e os efeitos do touro em serviço associado com a qualidade do sêmen e da libido. O modelo biológico total é recursivo, já que a data do parto anterior, em conjunção com o anestro pós-parto determina completamente a data do ciclo estral no corrente ano (MacNeil \& Newman, 1994). Assim, alguns estudos sobre a modelagem do DIAP têm observado efeitos significativos da duração da gestação (MacGregor \& Casey, 1999), do peso ao nascer ou sexo do bezerro (MacNeil \& Newman, 1994, Bergmann et al., 1998) além de outros fatores relacionados ao ciclo reprodutivo anterior, como peso ao nascer ou ao desmame (MacGregor \& Casey, 1999) e dias ao parto (Rege \& Famula, 1993, Bergmann et al., 1998) anteriores. A inclusão, no modelo, de fatores que são dependentes do parto anterior, reduz a amostra a registros das vacas que pariram no ano anterior, e a inclusão de fatores relacionados ao parto presente, como peso ao nascer do bezerro, impossibilita a inclusão das fêmeas não paridas na análise. Em uma análise que não considera estes fatores, como no presente estudo, supõem-se que eles estejam ocorrendo aleatoriamente nas subclasses estudadas, não influenciando as soluções dos fatores fixos de interesse.

Componentes de variância e parâmetros genéticos

As herdabilidades para o caráter DIAP (Tabela 2) foram baixas, além de associadas a erros-padrão muitas vezes da mesma magnitude do próprio parâmetro. Valores entre 0,02 a 0,09 foram relatados em grande parte dos trabalhos (Meyer et al., 1990, MacNeil \& Newman, 1994, Morris \& Cullen, 1994; Johnston \& Bunter, 1996; Gressler et al., 2000; Morris et al., 2000; Pereira et al., 2000). Quando os registros das fêmeas não paridas foram considerados nos arquivos conjuntos de vacas e novilhas, a herdabilidade do DIAP passou para 0,12 a 0,16 , com erros-padrão menores pelo maior número de registros, o que se explica pelo fato dos arquivos sem as fêmeas não paridas serem amostras mais selecionadas que quando se incluem os registros de todas aquelas que

R. Bras. Zootec., v.31, n.4, p.1715-1725, 2002 
foram escolhidas para reprodução (mesmo das inférteis), ou seja, a observação de caracteres de reprodução somente das fêmeas férteis, que parem pelo menos uma vez, contribui para mascarar a variabilidade das características e as diferenças genéticas entre os animais (Notter, 1988, Meyer et al., 1990). Quando esta variabilidade é re-introduzida, designando-se penalidades às fêmeas que não pariram, as diferenças genéticas entre os animais ficam evidentes. Este aumento na herdabilidade não foi observado no arquivo das novilhas, cujas estimativas de herdabilidade foram iguais, considerando ou não as fêmeas não paridas. Semelhantemente, Pereira et al. (2000) não observaram qualquer alteração na estimativa de herdabilidade quando os registros das fêmeas não paridas foram considerados na análise, através da penalização pelo método de 21 dias descrito anteriormente.

Os valores genéticos médios anuais, obtidos em análises univariadas, para o DIAP de novilhas (Figura 2) e de vacas e novilhas (Figura 3), ambos incluindo os registros das fêmeas não paridas, mostraram tendência de declínio no decorrer dos anos, nos três rebanhos $(-0,09 \pm 0,02 \mathrm{dia} /$ ano nas novilhas e $-0,24 \pm 0,04$ dia/ano nas vacas e novilhas), apesar da alta variação na resposta observada para este caráter. Deve ser ressaltado que a brusca diminuição do valor genético das fêmeas nascidas nos últimos anos do experimento pode ser simplesmente devido ao fato dessas fêmeas terem tido menor número de montas e, portanto, menor número de falhas que aquelas mais velhas. As soluções do efeito de ano de nascimento obtidas simultaneamente às soluções dos efeitos de animal na análise dos registros de vacas e novilhas, expressas como desvios do ano 1982, fornecem uma estimativa da tendência ambiental para DIAP (Figura 4) igual a $-0,84 \pm 0,32$ dia/ano. Ponzoni (1992) comenta que a escolha da data em que a estação de monta iniciou a cada ano pode contribuir para a mudança ambiental, e a interpretação biológica para tal tendência pode ser inválida. Na tentativa de minimizar este vício, ajustou-se um modelo considerando o efeito do dia da entrada na monta como covariável, e como este foi não significativo, as soluções para ano de nascimento confirmaram a tendência mostrada na Figura 4.

Observa-se a coerência da tendência genética com os resultados obtidos para o efeito de rebanho, e aqueles da interação rebanho $\mathrm{x}$ ano de nascimento, significativa $(\mathrm{P}<0,01)$ para as novilhas e não significativa $(\mathrm{P}=0,06)$ para vacas e novilhas (resultados não mostrados). MacNeil \& Newman (1994) relataram ganho genético praticamente nulo para dias ao parto das matrizes Hereford da Linha 1 de Montana, de 1940 a 1990, após 13 gerações de seleção, enquanto que os valores genéticos estimados para peso a desmama e peso a um ano de idade aumentaram, em média, 14,5 kg e 62,4 kg, respectivamente (MacNeil et al., 1992).

As estimativas de repetibilidade do DIAP (Tabela 2), ficaram entre os valores encontrados em outras publicações (Meyer et al., 1990, Rege \& Famula, 1993; Johnston \& Bunter, 1996; Morris et al., 2000; Pereira et al., 2000) e a inclusão das fêmeas não paridas teve efeito sobre estas estimativas.

Implicações

Apesar do caráter DIAP, ou equivalentemente o dia do parto no calendário juliano ser um dos caracteres mais simples de ser obtido (Ponzoni, 1992), considerando a complexidade da reprodução em fêmeas, os bancos de dados da maioria das associações de raças ou empresas privadas foi organizado de modo a ter registrado somente os nascimentos, isto é, registros provenientes das montas férteis, e não o registro de todas as montas, observações úteis para a inclusão das fêmeas não paridas nas análises. A solução para isso seria considerar a falta de registro em um ano, seguido de registro de parto em ano subseqüente, como uma monta sem sucesso, e assim incluir esta "falta de informação" nas avaliações genéticas. Ponzoni (1992) e Johnston \& Bunter (1996) defendem o uso do DIAP, incluindo as fêmeas não paridas, como o caráter que deve ser considerado nas avaliações genéticas nacionais, pelo fato de medir a taxa

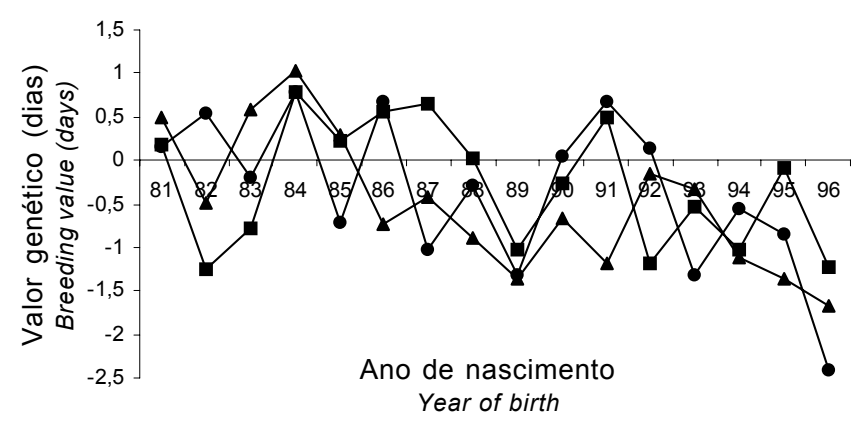

Figura 2 - Valores genéticos médios para dias ao parto da primeira monta, nos três rebanhos (controle $\mathbf{\square}$, seleção $\bullet$, tradicional $\mathbf{\Delta}$ ).

Figure 2 - Means of breeding values for days to calving for first breeding season, in the three lines (control $\mathbf{m}$, selection $\bullet$, traditional $\mathbf{\Delta}$ ). 


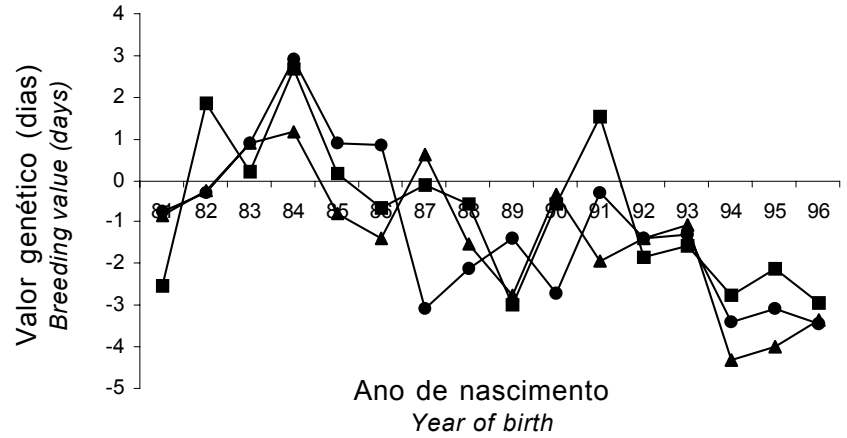

Figura 3 - Valores genéticos médios para dias ao parto de novilhas e vacas, nos três rebanhos (controle $\mathbf{n}$, seleção $\bullet$, tradicional $\mathbf{\Delta}$ ).

Figure 3 - Means of breeding values for days to calving for heifers and cows, in the three lines (control selection $\bullet$, traditional $\mathbf{\Delta}$ )

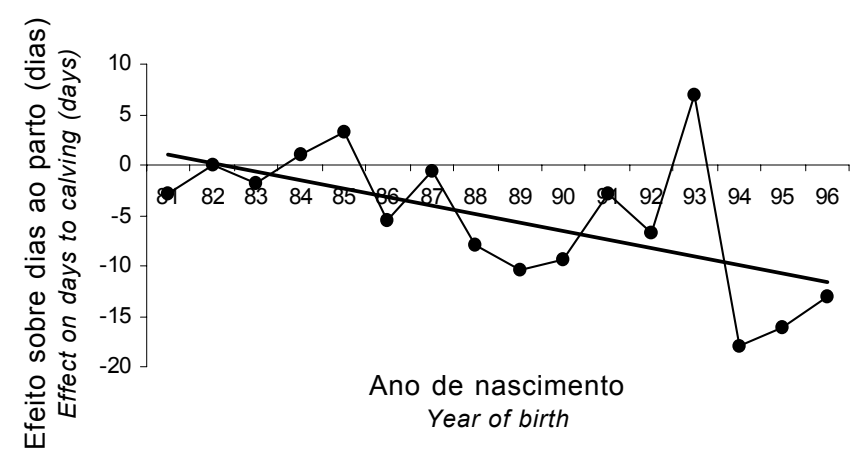

Figura 4 - Estimativa da tendência ambiental por ano de nascimento de novilhas e vacas para dias ao parto, expressas como desvio de 1982.

Figure 4 - Estimate of environmental trend, for year of birth of heifers and cows, in days to calving, shown as the deviation of 1982.

de prenhez, além de identificar as fêmeas com capacidade de emprenhar mais cedo na estação de monta. No Brasil, a avaliação genética de uma grande empresa privada de melhoramento genético já inclui o valor genético para dias ao parto das filhas nas avaliações genéticas de reprodutores Nelore (CFM, 1999).

Os resultados encontrados comparando o desempenho reprodutivo de fêmeas selecionadas para peso ao sobreano por 3,5 gerações de seleção (Razook et al., 1998), com fêmeas selecionadas para média deste peso, concordam com os trabalhos publicados na última década, tanto com dados experimentais
(Wolfe et al., 1990, Morris et al., 1992, MacNeil \& Newman, 1994, Parnell et al., 1994; Archer et al., 1998) como com dados de campo (Meyer et al., 1991; Johnston \& Bunter, 1996; Silva et al., 2002, Mercadante et al., 2000), indicando que a seleção para peso corporal não comprometeu o desempenho reprodutivo das fêmeas, mesmo estando os três rebanhos sob níveis nutricionais similares.

\section{Conclusões}

A seleção para peso corporal não acarretou diferenças no desempenho reprodutivo das fêmeas, mesmo sendo criadas em condições ambientais similares. Dias ao parto (considerando as fêmeas não paridas) pode ser incluído como um caráter de desempenho reprodutivo em avaliações genéticas, pois além de medir a taxa de prenhez, identifica as fêmeas com capacidade de emprenhar mais cedo na estação de monta.

\section{Literatura Citada}

ARCHER, J.A.; ARTHUR, P.F.; PARNELL, P.F. et al. Effect of divergent selection for yearling growth rate on female reproductive performance in Angus cattle. Livestock Production Science, v.57, p.33-40, 1998.

BAKER, R.L.; MORRIS, C.A.; JOHNSON, D.L. et al. Results of selection for yearling or 18-month weight in Angus and Hereford cattle. Livestock Production Science, v.29, p.277-296, 1991

BERGMANN, J.A.G.; GRESSLER, S.L.; PEREIRA, C.S. et al Avaliação de fatores genéticos e de ambiente sobre algumas características reprodutivas de fêmeas da raça Nelore em regime de estação de monta restrita. Arquivos Brasileiros de Medicina Veterinária e Zootecnia, v.50, p.633-645, 1998. BOLDMAN, K.G.; KRIESE, L.A.; VAN VLECK, L.D. et al. A manual for use for MTDFREML. A set of programs to obtain estimates of variance and covariance. Lincoln: Department of Agricultural/Agricultural Research Service, 1995. 120 p.

CFM Agro-pecuária. Sumário de Touros Nelore 1999. São José do Rio Preto: 1999. 54p.

CYRILLO, J.N.S.G.; RAZOOK, A.G.; FIGUEIREDO, L.A. et al. Efeitos da seleção para peso pós-desmame sobre medidas corporais e perímetro escrotal de machos Nelore de Sertãozinho (SP). Revista Brasileira de Zootecnia, v.29, p.403-412, 2000

DAVIS, G.P.; CORBET, N.J.; MACKINNON, M.J. et al. Response in female fertility and calf growth to selection for pregnancy rate in tropical beef cattle. Australian Journal of Agricultural Research, v.44, p.1509-1521, 1993.

GRESSLER, S.L.; BERGMANN, J.A.G.; PEREIRA, C.S. et al. Estudo das associações entre perímetro escrotal e características reprodutivas de fêmeas Nelore. Revista Brasileira de Zootecnia, v.29, p.427-437, 2000

HERD, R.M. Effect of divergent selection for yearling growth rate on the maintenance feed requirements of mature Angus cows. Livestock Production Science, v.41, p.39-49, 1995. 
JOHNSTON, D.J.; BUNTER, K.L. Days to calving in Angus cattle: genetic and environmental effects, and covariances with other traits. Livestock Production Science, v.45, p.13-22, 1996.

KOCH, R.M.; GREGORY; K.E., CUNDIFF, L.V. Critical analysis of selection methods and experiments in beef cattle and consequences upon selection programs applied. In: WORLD CONGRESS ON GENETICS APPLIED TO LIVESTOCK PRODUCTION, 2., 1982, Madrid. Proceedings.... Madrid: WCGALP, 1982. v.5, p.514-526.

LITTEL, R.C.; MILLIKEN, G.A.; STROUP, W.W. et al. SAS system for mixed models. Cary: SAS Institute Inc., 1996. $633 \mathrm{p}$.

MACGREGOR, R.G.; CASEY, N.H. Evaluation of calving interval and calving date as measures of reproductive performance in a beef herd. Livestock Production Science, v.57, p.181-191, 1999.

MACNEIL, M.D.; NEWMAN, S. Genetic analysis of calving date in Miles City Line 1 Hereford cattle. Journal of Animal Science, v.72, p.3073-3079, 1994.

MACNEIL, M.D.; URICK, J.J.; NEWMAN, S. et al. Selection for postweaning growth in inbred Hereford cattle: the Fort Keogh, Montana Line 1 example. Journal of Animal Science, v.70, p.723-733, 1992.

MERCADANTE, M.E.Z.; LÔBO, R.B.; OLIVEIRA, H.N. Estimativas de (co)variâncias entre características de reprodução e de crescimento em fêmeas de um rebanho Nelore. Revista Brasileira de Zootecnia, v.29, p.997-1004, 2000.

MEYER, K.; HAMMOND, K.; MACKINNON, M.J. et al. Estimates of covariances between reproduction and growth in Australian beef cattle. Journal of Animal Science, v.69, p.3533-3543, 1991.

MEYER, K.; HAMMOND, K.; PARNELL, P.F. et al. Estimates of heritability and repeatability for reproductive trait in Australian beef cattle. Livestock Production Science, v.25, p.15-30, 1990.

MORRIS, C.A.; BAKER, R.L.; HUNTER, J.C. Correlated responses to selection for yearling or 18-month weight in Angus and Hereford cattle. Livestock Production Science, v.30, p.33-52, 1992.

MORRIS, C.A.; CULLEN, N.G. A note on genetic correlations between pubertal traits of males or females and lifetime pregnancy rate in beef cattle. Livestock Production Science, v.39, p.291-297, 1994.

MORRIS, C.A.; WILSON, J.A.; BENNET, G.L. et al. Genetic parameters for growth, puberty, and beef cow reproductive traits in a puberty selection experiment. New Zealand Journal of Agricultural Research, v.43, p.83-91, 2000.

NOTTER, D.R. Evaluating and reporting reproductive traits. In: BEEF IMPROVEMENT FEDERATION, 20., 1988, Albuquerque, New Mexico. Proceedings... Albuquerque, New Mexico: BIF, 1988. p.21-42.

PARNELL, P.F. The consequences of selection for growth rate in beef cattle. Proceedings of Australian Society of Animal Production, v.20, p.17-26, 1994.

PARNELL, P.F.; ARTHUR, P.F.; BARLOW, R. Direct response to divergent selection for yearling growth rate in Angus cattle. Livestock Production Science, v.49, p.297-304, 1997.
PEREIRA, E.; ELER, J.P.; FERRAZ, J.B.S. Correlação genética entre perímetro escrotal e algumas características reprodutivas na raça Nelore. Revista Brasileira de Zootecnia, v.29, p.1676-1683, 2000.

PONZONI, R.W. Which trait for genetic improvement of beef cattle reproduction: calving rate or calving day? Journal of Animal Breeding and Genetic, v.109, p.119-128, 1992.

PONZONI, R.W.; GIFFORD, D.R. Reproductive and some peri-natal variables in a mixed breed beef cattle herd. Journal of Animal Breeding and Genetic, v.111, p.52-64, 1994.

RAUW, W.M.; KANIS, E.; NOORDHUIZEN-STASSEN, E.N. et al. Insiderable side effects of selection for high production efficiency in farm animals: a review. Livestock Production Science, v.56, p.15-33, 1998.

RAZOOK, A.G.; FIGUEIREDO, L.A.; BONILHA NETO, L.M. et al. Intensidades de seleção e respostas direta e correlacionadas em 10 anos de progênies de bovinos das raças Nelore e Guzerá selecionadas para peso pós-desmame. Boletim de Indústria Animal, v.50, p.147-163, 1993.

RAZOOK, A.G.; FIGUEIREDO, L.A.; BONILHA NETO, L. et al. Selection for yearling weight in Nelore and Guzera zebu breeds: selection applied and response in 15 years of progeny. In: WORLD CONGRESS ON GENETICS APPLIED TO LIVESTOCK PRODUCTION, 6., 1998, Armidale, Australia. Proceedings... Armidale: WCGALP, 1998. p.133-136.

RAZOOK, A.G.; FIGUEIREDO, L.A.; CYRILLO, J.N.S.G. et al. Prova de ganho de peso. Normas adotadas pela Estação Experimental de Zootecnia de Sertãozinho. Nova Odessa, Instituto de Zootecnia, 1997. 33p. (Boletim Técnico, 40).

REGE, J.E.O.; FAMULA, T.R. Factors affecting calving date and its relationship with production traits of Hereford dams. Animal Production, v.57, p.385-395. 1993.

SAS INSTITUTE. SAS/STAT User's guide. Version 8. Cary: 2000.

SCHOLTZ, M.M.; ROUX, C.Z. Correlated responses to selection for growth, size and efficiency. In: WORLD CONGRESS ON SHEEP AND BEEF CATTLE BREEDING, 2., 1984, Pretoria, South Africa. Proceedings... Pretoria: WCSBCB, 1984. p.433-443.

SILVA, A.M.; ALENCAR, M.M.; FREITAS, A.R. et al. Herdabilidades e correlações genéticas para peso e perímetro escrotal de machos e características reprodutivas e de crescimento de fêmeas, na raça Canchim. Revista Brasileira de Zootecnia, v.29, p.2223-2230, 2000.

WOLFE, M.W.; STUMPF, T.T.; WOLFE, P.L. et al. 1990. Effect of selection for growth traits on age and weight at puberty in bovine females. Journal of Animal Science, v.68, p.1595-1602.

Recebido em: 31/07/01 Aceito em: 17/04/02 